\title{
Screening to Detect Hip and Groin Problems in Elite Adolescent Football (Soccer) Players - Friend or Foe?
}

\author{
Matthew D DeLang, PT, DPT, CSCS $^{1}$ a J. Craig Garrison, PhD, PT, SCS, ATC ${ }^{2}$, Kristian Thorborg, PT, PhD ${ }^{3}$ \\ ${ }^{1}$ Right To Dream Academy, ${ }^{2}$ Texas Health Sports Medicine, ${ }^{3}$ Sports Orthopedic Research Center Copenhagen (SORC-C), Department of Orthopedic \\ Surgery, Amager-Hvidovre Hospital; Department of Clinical Medicine, University of Copenhagen \\ Keywords: groin pain, monitoring system, early detection, sports medicine
}

https://doi.org/10.26603/001c.21525

\section{International Journal of Sports Physical Therapy}

Vol. 16, Issue 2, 2021

Injury prevention strategies in team settings should not overlook early detection and secondary prevention. Monitoring systems may be an effective approach to detect common and troublesome injuries, such as hip and groin pain in football (soccer) players. The purpose of this International Perspective is to share our experiences with monitoring hip and groin pain in youth academy football and discuss challenges that surfaced. We consider why players may not accurately report pain, their perceptions of groin pain, and whether all groin pain is clinically meaningful.

\section{Level of Evidence}

5

Sports medicine staffs working in teams settings should emphasize injury risk reduction. Primary prevention strategies such as FIFA 11+, Nordic hamstring exercise, and Copenhagen adductor exercise programs are successful for hip and groin pain. ${ }^{1,2}$ Secondary prevention has been suggested using monitoring systems in Australia and Scandinavia. This approach to early detection can combat troublesome hip and groin pain in athletes, ${ }^{3-5}$ but it is not without challenges.

Hip and groin pain are common in elite male football (soccer) players, ${ }^{6,7}$ and recent authors have also shown high rates in youth players. ${ }^{3,8}$ In a retrospective anonymous survey after pre- and early season, ${ }^{9}$ we observed $77 \%$ of US academy football players had experienced groin pain of varying intensities (Figure 1).

We had simultaneously implemented a weekly monitoring system to screen groin pain in the academy. Surprisingly, the retrospective survey results did not align with weekly check-ins, where only $58 \%$ of players reported pain. We began to question if players were under-reporting their hip and groin symptoms to our medical personnel (physical therapists, athletic trainers, team doctors) at weekly checkins. The purpose of this International Perspective is to share our experiences with monitoring hip and groin pain in youth academy football and discuss challenges that surfaced. This may have implications for worldwide adoption

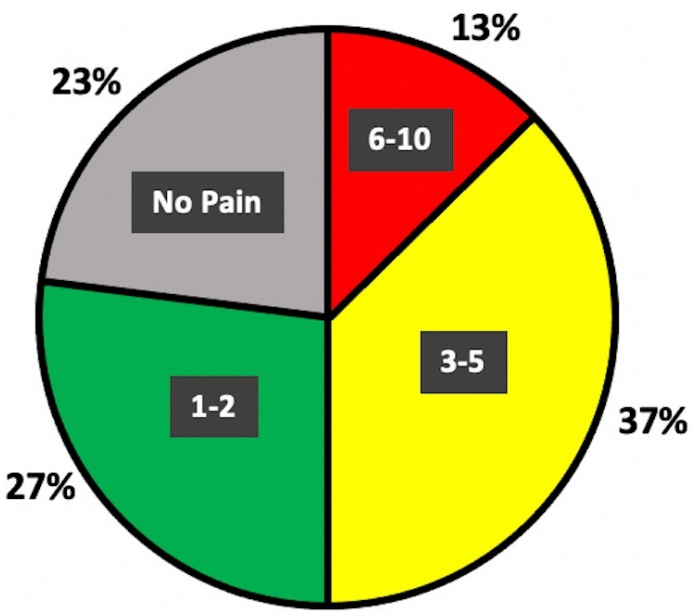

Figure 1: Reports of groin pain intensity (numeric pain rating scale; $0=$ no pain, $10=$ severe pain) during the first twelve weeks of pre-season and early-season in academy football players $(n=78)$.

of secondary prevention strategies in elite youth football.

One challenge for monitoring systems is relying on players themselves to accurately report their symptoms. Not

\footnotetext{
a Corresponding Author:

Matthew D. DeLang

Right To Dream Academy

Old Akrade, Eastern Region, Ghana

+2330201116199

mddelang@gmail.com
} 
only did we identify that groin pain was under-reported during weekly check-ins compared to retrospective reports (58\% at weekly check-ins versus $77 \%$ at the 12 -week retrospective survey), but we also retrospectively asked players why some might under-report pain. Players' perceptions of the monitoring system were gauged by asking if any of the following reasons might lead to under-reporting: 1) fear that reporting pain would restrict participation, 2) believing groin pain is normal, 3) not wanting to report in front of teammates, 4) reporting pain would make others question that player's toughness, or 5) a free-text option. A staggering $41 \%$ of academy players feared that reporting pain would lead to medical personnel preventing them from training/playing. In hindsight, we see that temporarily sidelining players for a thorough clinical examination backfired in relation to the trust between medical personnel and players. This rationale for players' under-reporting makes sense: if they perceive reporting symptoms as a threat to their participation, they will be less likely to report pain. The lesson is learned - medical personnel must consistently build trust with players and provide additional education to enhance player adherence to a monitoring system.

Another interesting lesson was how football players perceive groin pain. The survey revealed that $26 \%$ of players believed hip and groin pain was normal. Additionally, these statements appeared via free-text response: "my discomfort hasn't affected me", "it didn't hurt that bad”, and "pain wasn't bad enough to report”. Quite a few players did not find groin pain to be particularly concerning. This provided an opportunity to better understand our athletes. Players may perceive groin pain as a normal byproduct of football participation, and medical personnel must decide which pain is concerning and which is not. Transparent education and communication between all stakeholders (medical, coaches, players) may help understand the intention of the monitoring system: to collect information, detect problems, and intervene appropriately with secondary prevention and treatment.

Upon establishing consistent and accurate reporting with the athletes, a monitoring system provides the means to implement secondary injury prevention. Ideally, medical personnel would intervene when groin pain is meaningful (i.e., at-risk for worsening severity, time-loss, and high injury burden), while also supporting participation when it isn't (i.e., normal soreness and fatigue). Herein lies another big challenge: what are ways to differentiate between meaningful and meaningless groin pain? The Doha Agree- ment ${ }^{10}$ clinical taxonomy provides a standardized framework to classify groin pain. Perhaps monitoring pain using this taxonomy, coupled with symptom duration and intensity, can help medical personnel pinpoint when pain that spreads to another location, persists over time, or reaches higher intensity becomes more concerning.

The authors of this perspective have identified challenges encountered during hip and groin monitoring, including why athletes might under-report pain, whether all groin pain is meaningful, and knowing how and when to intervene. Researchers implementing monitoring systems should be encouraged to share experiences to add to the global perspectives of monitoring athletes for early detection. We believe improving communication between all stakeholders promotes shared decision-making for safe participation and optimal performance, and that trust is essential so that players and medical personnel can profit from the monitoring system. Learning how and when to intervene will improve injury risk reduction strategies in team sports settings. Presently, this may not be as simple as it sounds, as further development around such strategies and their implementation may be needed before athletes view close monitoring as a "friend".

\section{COMPETING INTERESTS}

None.

\section{PATIENT CONSENT FOR PUBLICATION}

The survey referred to in this paper was approved by the University of Texas Southwestern Institutional Review Board and all academy players completed informed consent and assent. Those under the age of 18 were signed by a parent or guardian.

\section{ACKNOWLEDGEMENTS}

The authors would like to thank the academy medical staff at FC Dallas, including J Rios and D Woodhouse, for their assistance and commitment to this project.

Submitted: February 01, 2021 CDT, Accepted: March 01, 2021 CDT 


\section{REFERENCES}

1. Ishøi L, Krommes K, Husted RS, Juhl CB, Thorborg K. Diagnosis, prevention and treatment of common lower extremity muscle injuries in sport - grading the evidence: A statement paper commissioned by the Danish Society of Sports Physical Therapy (DSSF). Br J Sports Med. 2020;54(9):528-539. doi:10.1136/bjsport $\underline{\mathrm{s}-2019-101228}$

2. Thorborg K, Krommes KK, Esteve E, Clausen MB, Bartels EM, Rathleff MS. Effect of specific exercisebased football injury prevention programmes on the overall injury rate in football: A systematic review and meta-analysis of the FIFA 11 and $11+$ programmes. Br J Sports Med. 2017;51(7):562-571. do i:10.1136/bjsports-2016-097066

3. Wollin M, Thorborg K, Welvaert M, Pizzari T. Inseason monitoring of hip and groin strength, health and function in elite youth soccer: Implementing an early detection and management strategy over two consecutive seasons. J Sci Med Sport.

2018;21(10):988-993. doi:10.1016/j.jsams.2018.03.00 $\underline{4}$

4. Wörner T, Thorborg K, Eek F. Five-second squeeze testing in 333 professional and semiprofessional male ice hockey players: How are hip and groin symptoms, strength, and sporting function related? Orthop J Sports Med. 2019;7(2):232596711982585. doi:10.1177/ $\underline{2325967119825858}$
5. Thorborg K, Branci S, Nielsen MP, Langelund MT, Hölmich P. Copenhagen five-second squeeze: A valid indicator of sports-related hip and groin function. $\mathrm{Br}$ J Sports Med. 2017;51(7):594-599. doi:10.1136/bjsport $\underline{\mathrm{s}-2016-096675}$

6. Thorborg K, Rathleff MS, Petersen P, Branci S, Hölmich P. Prevalence and severity of hip and groin pain in sub-elite male football: A cross-sectional cohort study of 695 players. Scand J Med Sci Sports. 2017;27(1):107-114. doi:10.1111/sms.12623

7. Harøy J, Clarsen B, Thorborg K, Hölmich P, Bahr R, Andersen TE. Groin problems in male soccer players are more common than previously reported. Am J Sports Med. 2017;45(6):1304-1308. doi:10.1177/03635 $\underline{46516687539}$

8. DeLang MD, Garrison JC, Hannon JP, et al. Short and long lever adductor squeeze strength values in 100 elite youth soccer players: Does age and previous groin pain matter? Phys Ther Sport. 2020;46:243-248.

9. Esteve E, Rathleff MS, Hölmich P, et al. Groin problems from pre- to in-season: A prospective study on 386 male Spanish footballers. Res Sports Med. 2020;14:1-7. doi:10.1080/15438627.2020.1860044

10. Weir A, Brukner P, Delahunt E, et al. Doha agreement on terminology and definitions of groin pain in athletes. Br J Sports Med. 2015;49(12):768-774. doi:10.1136/bjsports-2015-0948 $\underline{69}$ 